\title{
Size and Defect related Broadening of Photoluminescence Spectra in ZnO:Si Nanocomposite Films
}

\author{
Shabnam ${ }^{a}$, Chhaya Ravi Kant ${ }^{a}$ and P. Arun ${ }^{b *}$ \\ ${ }^{a}$ Department of Applied Sciences, \\ Indira Gandhi Institute of Technology, \\ Guru Gobind Singh Indraprastha University, \\ Delhi 110 006, India. \\ ${ }^{b}$ Department of Physics \& Electronics, \\ S.G.T.B. Khalsa College, \\ University of Delhi, Delhi - 110 007, India
}

October 29, 2018

\begin{abstract}
Nanocomposite films of Zinc Oxide and Silicon were grown by thermal evaporation technique using varying ratios of $\mathrm{ZnO}: \mathrm{Si}$ in the starting material. Analysis reveal the role of $\mathrm{ZnO}$ and amorphous silicon interface in contributing to relatively less common blue photoluminescence emissions (at $\sim 400$ and $470 \mathrm{~nm}$ ). These blue peaks are observed along with the emissions resulting from band edge transition (370nm) and those related to defects $(522 \mathrm{~nm})$ of $\mathrm{ZnO}$. Careful analysis shows that along with the grain size of $\mathrm{ZnO}$, a suitable compositional ratio (of $\mathrm{ZnO}$ to silicon) is critical for the coexistence of all the four peaks. Proper selection of conditions can give comparable photoluminescence peak intensities leading to broadband emission.
\end{abstract}

Keywords Nano-composites, Nanostructures, Photoluminescence, Oxides

*email:arunp92@physics.du.ac.in, Telephone:091 011 29258401, Fax: 09101127666220 


\section{Introduction}

Recent research in Material Science is directed along the lines of tailoring material properties as per requirement. In this direction both materials in nano regime and in composite state has drawn attention. Interest hence has also been developed in materials in nanocomposite state. Nanocomposites by defintion refer to the class of composite materials wherein atleast one of the constituents exists in the nanometre range. Research on $\mathrm{ZnO}$ based nanocomposites has been a front-runner in recent times, especially in the field of white light emitting devices (LED) [1, 2]. These nanocomposites enjoy two-fold advantage over $\mathrm{ZnO}$ nanomaterials. While a carefully selected host material can result in broadening of light emission, it also gives additional stabilty to the $\mathrm{ZnO}$ film and prevents agglomeration of the grains. Of all the possible hosts, the research is mainly centred around the use of silicon and its varied complexes $[3,4,5,6,7,8$. This is because of the key position of silicon in the microelectonic industry and its abiliy to emit in the red region in the nanophase.

In quest for obtaining white light, Klason et al 9 have tried depositing n-type $\mathrm{ZnO}$ nanorods on p-Si, which emitted white light under forward biasing. Depositing of $\mathrm{ZnO}$ nanoparticles in porous silicon (po-Si) has also been reported by Bo et al [10] and Mehra et al [11]. Though the intensity of luminescence observed in Bo's work was high, use of porous Si substrate itself suffers from the demerit of being prone to quick oxidation and requires tedious method of production. Peng [12] and Pal [13] had studied the effect of varying ZnO to silicon content. Peng et al [12] were successful in obtaining white light from RF sputtered films with the lowest $\mathrm{ZnO}$ content ( $26 \%$ of $\mathrm{ZnO})$. By considering the role of heterogeneous boudaries and taking into account the depletion width thus developed, they explained the maximum obtained intensity of the blue photoluminescence (PL) peak observed in the samples with lowest Zinc Oxide nanoparticles. In a later work [14 they also discussed the color chromaticity dependance on the dot diameter. With their work they were able to prove that the neighbourhood and the size of the $\mathrm{ZnO}$ grains are both important for the efficient white light production. Using a similar method of fabrication Pal et al tried dispersing Si atoms in $\mathrm{ZnO}$ matrix. The samples were then annealed at high temperature. An increase in PL intensity was observed upon annealing and with decrease of $\mathrm{ZnO}$ content in the films. Unlike the PL behavior reported by Peng, the intensity in Pal's samples decreased with further lowering of $\mathrm{ZnO}$ content of the films. Thus it becomes clear that the compositional ratio of $\mathrm{ZnO}$ to Silicon which inturn means the neighbourhood offered to $\mathrm{ZnO}$ nanoparticles is deterimental in bringing desired changes in optical properties of the nanocomposites and should be given its due concern. Nanocomposites based on ZnO and Si have been fabricated by sol gel [15, 16, 17, 18, 19, RF sputtering [20, 21, 22, 23, pulsed laser deposition [24, via chemical routes [25, 26, 27, 28, reverse micelle method [29] and their optical properties studied. These research calls for more systematic work to optimize the parameteres for tailoring the properties and for a better understanding of interface and defect mechanism of $\mathrm{ZnO}: \mathrm{Si}$ nanocomposites

Hence to this effect, we have started systematic study of Zinc Oxide grains in nano regime embedded in amorphous Silicon matrix. In our earlier work, [30] we had demonstrated that these films emitted light in the visible region, namely in UV, blue, green and red regions. To investigate the tailoring of our samples' properties, we annealed our samples (post deposition) and reported broadening of photoluminescence emissions. Not only this, the intensity of the annealed samples increased manifold [31. In this manuscript we report the role of varying ratio of $\mathrm{ZnO}: \mathrm{Si}$ (in starting material) on the photoluminescence.

\section{$2 \quad$ Experimental Details}

The films of $\mathrm{ZnO}: \mathrm{Si}$ nanocomposites studied in this work were fabricated by thermally evaporating a mixture of powdered $\mathrm{ZnO}$ and n-Silicon. Vacuum of the order of $10^{-6}$ Torr was created in the deposition chamber of a Hind High Vac (Bengaluru), Thermal evaporation coating unit, Model 12A4D. The deposition was carried out on microcopic glass substrates maintained at room temeprature. To prevent the flying off, of starting material powdered $\mathrm{ZnO}$ and $\mathrm{Si}$, was pelletized by applying a pressure of $60 \mathrm{kN}$. ZnO used in this study was $(99.99 \%)$ pure and purchased from Merck (Mumbai). The mixture was prepared by mixing $\mathrm{ZnO}$ and Silicon in the proportions 
of 1:1, 1:2, 1:3, 2:3 and 2:5 (by weight). Films of these composition are hereafter referred to as sample (a), (b), (c), (d) and (e), respectively. We have restricted the present study to samples of $600 \AA$ thickness. The samples discussed in this work are as grown, not subjected to any post deposition heat treatment.

The structural studies of the surface is measured by Pananalytical PW3050/60 Grazing Incidence angle X-Ray Diffractometer (GIXD) and that of the bulk region by Philips PW 3020 X-Ray Diffractometer (XRD). XRay Photoelectron Spectroscopy (XPS) was performed with Perkin-Elmer X-ray Photo-electron Spectrometer (Model 1257) with Mg K $\alpha$ (1254 eV) X-ray source. Photoluminescence (PL) scans were recored on Jobin Yvon spectroscopes respectively. Renishaw's "Invia Reflex" Raman spectroscope was used for measurements. The surface morphology and texture of the as grown nanocomposite films were studied using the Scanning Electron Microscope (SEM), atomic force microscopy (AFM)- NTEGRA NS-150 and Transmission Electron Microscope (TEM). In the following section we enlist the results of the various analysis done on our samples.

\section{Results and Discussions}

\subsection{X-Ray Diffraction, Chemical Composition \& Morphological Studies}

The nanocomposite films were fabricated by co-evaporating Zinc Oxide with Silicon. The melting point of silicon is $1410^{\circ} \mathrm{C}$ at atmospheric pressure while that of Zinc Oxide is about $1975^{\circ} \mathrm{C}$. However, the melting point of Zinc Oxide is known to reduce in the presence of group-IV elements facilitating its thermal evaporation at a lower temperature [32, 33, 34]. Both these materials have diverse melting points which could result in multilayered structure rather than a homogeneous nanocomposite film. The possibility of formation of multilayered structure was overruled by IR analysis of the films which was reported in our earlier work [30].

We have further tried to resolve this question using grazing incidence angle X-Ray diffraction studies (GIXD). At critical incidence angle, usually lying between $1.5^{\circ}$ and $0.005^{\circ}$, the X-ray beam gets totally reflected from the surface. Infact below the critical angle, the reflected beam is allowed to penetrate only upto few nanometres [35, 36, 37, 38. This ensures X-Ray is being diffracted from the surface layer. Figure 1A exhibits the nature of the diffraction pattern obtained for films of the same thickness but varying ratios of Zinc Oxide to Silicon. These X-Ray diffraction pattern show prominent peaks at $2 \theta=43^{\circ}, 36^{\circ}$ and $38^{\circ}$. These peaks correspond to pure Zinc (ASTM Card No-4-831). The $36^{\circ}$ peak can possibly be unresolved peak of Zinc and Zinc Oxide (ASTM Card No-1451). The existence of elemental Zinc as shown by GIXD would imply that the surface of our films have metallic Zinc. This may not be the case within the surface of the films. To investigate this we have also studied the same samples using $\theta-2 \theta \mathrm{X}$-Ray diffraction studies. The results are shown in figure 1B. Barring sample (a) corresponding to the 1:1 ratio film, the X-Ray diffraction pattern of all the other samples were unmarked. This did not come as a surprise since the grain size of $\mathrm{ZnO}$ in nanocomposite films of $600 \AA$ is too small to result in X-Ray diffraction [30, 39.

In short, our X-Ray analysis suggests formation of pure Zinc on the surface of nanocomposite bulk, where Zinc Oxide is embedded within the matrix of Silcon. To investigate the depth to which elemental Zinc exist, we have analysed sample (a) using XPS. XPS scans were made at the surface and periodically repeated after removing layers of $100 \AA$ by ion milling. Figure $2 \mathrm{~A}$ shows the Zinc $1022 \mathrm{eV}$ peak associated with it $2 \mathrm{p}_{3 / 2}$ orbital. Figure 2A compares the Zinc peaks as scanned at depths of 100, 200,300, 400 and $500 \AA$. These are single peaks which show a shift to lower energy side with increasing depth. However, as can be seen from Figure 2B, the Zinc peak from the surface layer can be deconvoluted into two peaks. The peaks are separated by $\sim 1 \mathrm{eV}$. The $1021 \mathrm{eV}$ is associated with free Zinc or Zinc atoms which are not in bonding with any other species 40, 41, 42, 43, 44, while the $1022 \mathrm{eV}$ peak shows that there are sizeable amount of Zinc atoms that present in bonding with oxygen, existing as Zinc Oxide [40, 41]. To colloborate this, we have also scanned the layers for oxygen.

Figure $2 \mathrm{C}$ compares the oxygen peaks along the thickness of the film. Here also, oxygen seems to exist in two chemical compositional states as is indicated by the deconvoluted peaks shown in the figure. The only possibility is oxygen in bonding with Zinc and Silicon present in the film. The $530.5 \mathrm{eV}$ peak of the surface 
layer, corresponds to oxygen in bonding with Zinc, confirming that the surface of sample (a) has Zinc and Zinc Oxide. A close look of Figure 2C, reveals that the oxidation of silicon at the surface has not passivated the surface and protected the inner layers completely, as can be understood by the presence, abid diminishing contribution of the $532 \mathrm{eV}$ peak. This could be because the sample in question is of small thickness and the XPS scanning was done after a long time from its date of fabrication. Figure 2D exhibiting Si XPS peaks, also confirms the existence of Silicon and Silicon dioxide in the film with the deconvoluted peaks clearly observable through the entire thickness of the sample (a). This establishes that our film contain Zinc Oxide embedded in a matrix of Silicon and Silicon Dioxide with elemental Zinc at the surface. However, it must be emphasised again that the occurrence of silicon dioxide only took place after a long time from the sample's fabrication time.

Before proceeding it is worth mentioning again that the Zinc XPS peak corresponding to atoms in bonding with oxygen shifts with their position along the depth of the film. Figure 3 shows the near linear trend with this peak moving from $1022.4 \mathrm{eV}$ at the surface to $1021.8 \mathrm{eV}$ within the film. We beleive this shift in peak as you go within the thickness of the film is due to the chemical species and it's relative quantity existing around the $\mathrm{ZnO}$ molecules. Figure 3B clearly shows increase in relative Zinc Oxide as you move deeper into the film. It should be noted that our starting material for sample (a) had $50 \% \mathrm{ZnO}$ however as can be anticipated, the film's bulk only has 24-30\% ZnO. Figure 3C combined the best fit curves of Figure 3A and 3B and projects variation in peak position as $\mathrm{ZnO}$ percentage varies. Larger amount of Silicon seems to shift the $\mathrm{ZnO}$ peak to the higher energy side. This is the precise influence we are investigating and hope would assist in tailoring the optical properties of these nanocomposites. Also, we expect the same trend of Figure 3C for samples prepared with differnt $\mathrm{ZnO}$ presence. We are in process of studying samples with different starting ratios, same thickness and verifying this.

TEM micrograph (Figure 4) of sample (c) shows existence of two phases. The clusters of $\mathrm{ZnO}$ dispersed in a matrix of amorphous Silicon. The inset shows image taken in dark field which brings better contrast. For studying the film-morphology we carried out AFM analyis on the sample surface. The AFM images of the samples (a) and (b) are shown in Figure 5A and 5B, respectively. The AFM images reveal uniform films with particles ranging in nanometre. However, while discussing the X-Ray analysis results, we have clearly established the existence of elemental Zinc on the surface of the films hence, the observed grains and measured grain size are not typically of $\mathrm{ZnO}$. Hence, the grain size of $\mathrm{ZnO}$ was also calculated using the Debye-Scherrer formula [45] from the $36^{\circ}$ peak of GIXD. The grain size of $\mathrm{ZnO}$ was found to lie between 8.8 to $23 \mathrm{~nm}$ for our samples.

Along with the nature of Zinc Oxide's neighbouring chemical composition and it's grain size, it is understood that the strain acting on Zinc Oxide lattice also contributes to its optical properties. The $36^{\circ} \mathrm{ZnO}$ peak in XRD is only observed in one sample (that of ' $a$ ', see Figure 1B). There is a distinct right shift in the observed peak position with respect to the peak position reported for a single crystal. This shift is indicative of a tensile stress. We have calculated the strain from which stress can be calculated [45. Strain is usually evaluated using 45 .

$$
\sigma=\frac{d_{A S T M}-d_{o b s}}{d_{A S T M}}
$$

where $d_{\text {ASTM }}$ is the ' $d$ ' spacing for the single crystal structure and $d_{\text {obs }}$ is that for the sample in question. Using equation (1) the strain within the bulk of sample (a) is found to be $3.054 \times 10^{-3}$. As expected, this is different from strain acting on the surface, which was found to be $7.372 \times 10^{-3}$. However, since the order is same we can consider strain acting on surface is representative of that in the bulk. The surface strain was found to be decreasing with decreasing $\mathrm{ZnO}$ presence in the films. The tensile strain detected is due to the defects caused by vacancies [46]. This can also be appreciated from Raman spectra. Hence, we analysed our samples using Raman Spectra. The results of our study is discussed below. 


\subsection{Raman Spectra}

The Raman spectra were taken in standard back scattering geometry using $\mathrm{Ag}^{2+}$ laser for excitation. Figure 6 gives the Raman spectra for the sample (a), (d), (b), (e) and (c) respectively. Broad peaks were obtained from $300 \mathrm{~cm}^{-1}$ to $600 \mathrm{~cm}^{-1}$. These peaks were deconvoluted to give three peaks. We are confident about our deconvolution at $\sim 310 \mathrm{~cm}^{-1}$ and $\sim 570 \mathrm{~cm}^{-1}$ because of the prominent shoulders they give. The $\sim 310 \mathrm{~cm}^{-1}$ peak corresponds to the LA mode of amorphous Silicon [30, 51]. The intensity and contribution of this peak to the Raman spectra is indicative of the amorphous Silicon in our samples. The large Silicon shown in Raman analysis which were carried out soon after the fabrication confirms our arguement that Silicon Oxide (shown in XPS) formed with time.

The $\sim 570 \mathrm{~cm}^{-1}$ corresponds to the $\mathrm{A} 1 \mathrm{LO}$ peaks of $\mathrm{ZnO}$ which is essentially associated with defect structures in $\mathrm{ZnO}$. This defect in literature has been associated to either Oxygen vacancies or Zinc interstitial defects [47. From our X-Ray analysis on we reported vacancies giving rise to tensile strain. Hence, we beleive this $570 \mathrm{~cm}^{-1}$ peak is due to Oxygen vacancies in our samples. This is ofcourse expected to influence the optical properties of the samples. The third peak is at $\sim 430 \mathrm{~cm}^{-1}$. Although the position of this deconvoluted peak may not be as reliable as the other peaks because of the lack of prominent shoulders, this can be ascribed as the peak of $\mathrm{ZnO}$. The peak at $430 \mathrm{~cm}^{-1}$ is of $\mathrm{E}_{2}^{\mathrm{high}}$ mode of Zinc Oxide, usually reported at $437 \mathrm{~cm}^{-1}$ [48, 49, 50] and possibly merged with amorphous Silicon that is expected at $470-510 \mathrm{~cm}^{-1}\left[51,52,53\right.$. The $\mathrm{E}_{2}^{\text {high }}$ peak is related to the vibration of the oxygen atom in the wurtzite structured $\mathrm{ZnO}$ lattice. The presence of this huge peak in all the samples further confirms the presence of $\mathrm{ZnO}$ in the bulk region of the films. The existence of $430 \mathrm{~cm}^{-1}$ reconfirms our claim of $\mathrm{ZnO}$ being embedded in the amorphous Silicon.

It is clear from the Raman Spectra that our nano-composite samples is not just $\mathrm{ZnO}$ clusters dispersed in a matrix of amorphous Silicon but additionally the $\mathrm{ZnO}$ cluster is a homogeneous mix of lattice with perfect wurzite crystallinity and that with oxygen defects. Considering that the optical properties of $\mathrm{ZnO}$ strongly depends on it's structure giving PL emission at 520 or 370nm depending on existence of defects or good crystallinity, existence of both phases in our sample should result in both peaks occuring in our PL. Thus, a suitable ratio of the contents of these two phases should give rise to a broad emission in the PL. Before looking at PL, we plot the ratio of area enclosed by the Raman peaks at $430 \mathrm{~cm}^{-1}$ and $560 \mathrm{~cm}^{-1}$ with respect to the content of $\mathrm{ZnO}$ in the film (fig 7). Figure 7 shows that the two areas are comparable in samples (b), (d) and (e). Hence, we expect the PL emission peaks at 370 and $520 \mathrm{~nm}$ to be comparable in these three samples. In our next section we shall investigate these issues.

\subsection{Photoluminescence}

To study how the above discussed strcutural and compositional behaviour manifests in the optical properties we have studied the PL spectroscopy of our samples using an excitation source of Xenon lamp. The PL was scanned in the range of $290-800 \mathrm{~nm}$ on excitation with wavelength of $270 \mathrm{~nm}$. Since the measurements were taken up without the use of filters, the presence of second harmonics at $540 \mathrm{~nm}$ is inevitable and can be seen as line spectra along with the PL peaks (Figure 8). The PL of samples (a), (b), (c) and (d) exhibit similar behavior with four prominent peaks. There is a sharp peak at $370 \mathrm{~nm}$ accompanied by broad peaks at $410 \mathrm{~nm}$, $470 \mathrm{~nm}$ and $522 \mathrm{~nm}$. The $370 \mathrm{~nm}$ is due to the radiation resulting from electron transistion across the band gap of Zinc Oxide [1, 2]. The 522nm is well documented [54, 55, 56] and is related to intra-band transistions, i.e. between levels created by defects within the band gap, caused by the Oxygen vacancies. As discussed in the Raman analysis, we had predicted broadening of emission spectra in samples (b), (d) and (e) because they had nearly equal amount of $\mathrm{ZnO}$ lattice with and without defects. The expected broadening is evident in our $\mathrm{PL}$ (fig 8).

Broadening is also assisted in our PL due to additional peaks at 410 and $470 \mathrm{~nm}$. The $414 \mathrm{~nm}$ peak have been attributed to inter-grain boundary defects [31 in $\mathrm{ZnO}$. However, over the large amount of samples we have studied we have observed that the 410 and $470 \mathrm{~nm}$ peaks appear only if the background is a-Si. These peaks 
were absent in samples with n-Si background [30]. Hence, we propose that these peaks are indeed influenced by the intergrain boundary and the background species rather than, if not only by the defects. To further our idea, we argue that if these peaks are contributed by interface and hence by the net surface area of the $\mathrm{ZnO}$ grains, then the peak intensity would be a result of the grain size and number of grains present. This ofcourse is the underlying principle of nanotechnology. Assuming the number of grains in the film $(\mathrm{N})$ to be proportional to the ratio of $\mathrm{ZnO}$ present in the starting material and using the grain size(R) evaluated using debye-Scherrer equation, we plotted a curve between the peak intensity of $465 \mathrm{~nm}$ with $\mathrm{R}^{2} \mathrm{~N}$ (fig 9). We find this to be linear suggesting its dependence on the interface of $\mathrm{ZnO}$ and amorphous Silicon.

Similar correlation is found among results of other analysis. For example, the 522nm PL peak arises due to oxygen vacancy defects. The defects also manifest as strain in the film as seen by the displacement of XRD peak from expected position. The perfect linear relation between the area of the green PL peak with strain in samples, (fig 9b) confirm our inferences. The omission of the $365 \mathrm{~nm}$ peak also shows proportionality with amount of wurtzite species present as seen from Figure 9(c) which plots area under 365nm peak of PL with respect to $438 \mathrm{~cm}^{-1}$ peak from Raman spectra. We can thus summarise that a sample with appropriate mixture of defects and defect free $\mathrm{ZnO}$, grown with a starting material of 1:2 $\mathrm{ZnO}$ to Silicon with grains in nano regime would give four emission peaks that overlap. The overlapping results in broadening best understood by a schematic shown in Figure 10.

\section{Conclusion}

We have fabricated nanocomposite films of $\mathrm{ZnO}$ and $\mathrm{Si}$ using thermal evaporation technique with clusters of $\mathrm{ZnO}$ dispersed in a matrix of amorphous Silicon. Depth profiling ESCA scans along with earlier IR studies confirm formation of our nano-composite films. The PL of the samples shows four prominent peaks in the bluegreen region, namely $\sim 400,470,370$ and $522 \mathrm{~nm}$. The latter peaks are attributed to emissions via transistions from the band edge and defect related peaks from $\mathrm{ZnO}$. On the other hand the 400 and $470 \mathrm{~nm}$ peaks exist because of the interface between $\mathrm{ZnO}$ grains and amorphous silicon. The interface increases with decreasing grain size and increasing number of $\mathrm{ZnO}$ grains. This was established from the observation of varying $\mathrm{PL}$ intensity with varying the $\mathrm{ZnO}: \mathrm{Si}$ compositional ratio and grain size. Samples with appropriate mixture of defects and defect free $\mathrm{ZnO}$ nanostuctures lead to broadening due to merging of peaks. We have found the 1:2 sample with maximum broadening. This sample with $22.4 \mathrm{~nm}$ grain size also gave rise to large $465 \mathrm{~nm}$ peak in PL indicating formation of large interfacial boundary. It thus proves, that not only the grain structure but also the interfacial boundaries and the nature of background species play a decisive role in equalizing the intensity accompanied by the broadening of the PL peaks. At present, we only observe a weak red emission and hope to control and improve the intensity of this peak. Enhancement and ultimately merging of red peak with the remaining emission spectra would estiblish $\mathrm{ZnO}: \mathrm{Si}$ nano-composites as potential candidate for the development of cost effective white light emitting diodes.

\section{Acknowledgment}

The resources utilized at Indian Institute of Technology, Delhi and Geology Department, University of Delhi is gratefully acknowledged. Also, the resources used at the Instrumentation Center and University Information Resource Center, Guru Gobind Singh Indraprasta University is also acknowledged. We also would like to express our gratitude to Dr. Kamal Sanan and Dr. Mahesh Sharma (both at National Physical Lab., Delhi) for carrying out the photoluminescence and XPS studies respectively. Authors PA and CRK are thankful to University Grants Commission (India) for financial assistance in terms of Major Research Award, F.No-33$27 / 2007$ (SR). 


\section{Figure Captions}

1. X-Ray Diffractograms of sample (a),(b),(c),(d) and (e) obtained by (A) GIXD and (B) $\theta-2 \theta$ technique.

2. (A) XPS $2 \mathrm{p}_{3 / 2}$ peaks of Zinc taken at various depth of same sample showing two species, (B) surface scan, (C) depth profile scans for Oxygen and (D) Silicon.

3. Variation of (A) Peak position of Zn with depth (B) Fraction of Zinc in bonding with Oxygen to amount of Silicon present along the thickness and (C) Peak position of Zinc in bonding with Oxygen to its fraction of presence.

4. TEM of sample (c). Inset shows dark field image of same sample taken at low magnification which clearly shows formation of two phase with $\mathrm{ZnO}$ clusters embedded in amorphous silicon.

5. AFM images of sample (a) and sample (b).

6. Raman spectra of sample (a), (d), (b), (e) and (c). Also seen are deconvoluted peaks assigned to amorphous silicon, wurtzite structure $\mathrm{ZnO}$ and with oxygen vacancies defects.

7. Relative presence of $\mathrm{ZnO}$ with oxygen vacancies to wurtzite structure $\mathrm{ZnO}$ (Area $560 \mathrm{~cm}^{-1} /$ Area $438 \mathrm{~cm}^{-1}$ from Raman spectra) for varying $\mathrm{ZnO}$ content in film.

8. PL of sample (a), (d), (b), and (c). Alongside the raw spectra are shown, deconvolution give 365, 400 and $465 \mathrm{~nm}$ between 300 and $480 \mathrm{~nm}$. Also green emission due to defects have been separated from $2^{\text {nd }}$ harmonic to show relative contributions.

9. The (A) increase in contribution of $465 \mathrm{~nm}$ PL peak with increasing $R^{2} N$, (B) linear relation in green $(522 \mathrm{~nm})$ emission with strain in film and inturn oxygen vacancies and $(\mathrm{C})$ co-relation in existence of wurtzite peak $\left(438 \mathrm{~cm}^{-1}\right.$ in Raman spectra) and blue emision (365nm peak of PL) in samples.

10. Schematic explaining broadening of $\mathrm{PL}$ in $\mathrm{ZnO}$ :Si nanocomposites and their individual contributions. 


\section{References}

[1] C. Jagadish and S. J. Pearton, "Zinc Oxide Bulk, Thin Films and Nanostructures, Elsevier Ltd.", 2006.

[2] U. Ozgur, Ya. I. Alivov, C. Liu, A. Teke, M. A. Reshchikov, S. Dogan, V. Avrutin, S.-J. Cho and M. Morkoc, J. Appl. Phys., 98 (2005) 041301.

[3] A.K.Das, P.Misra and L.M.Kukreja, J.Phys. D:Appl. Phys.,42 (2009) 165405.

[4] B.Yang, A.Kumar, H.Zhang, P.Feng, R.S.Katiyar and Z.Wang, J.Phys. D:Appl. Phys.,42 (2009) 045415.

[5] W.J.Shen, J.Wang, Q.Y. Wang, Y.Duan and Y.P. Zheng, J.Phys. D:Appl. Phys.,39 (2006) 269.

[6] E.Bohmer, F.Siebke and H.Wagner, Fresenius J.Anal.Chem, J.Phys. D:Appl. Phys.,358 (1997) 210.

[7] Q.Jiang, Zheng Ying Wu, Yi Meng Wang, Yi Cao, Chun Fang Zhou and Jian Hua Zhu, J.Mater.Chem., 16 (2006) 1536.

[8] P.Cheng, D.Li and D.Yang, Optics Express, Vol.16, No.12 (2008) 8898.

[9] P. Klason, P. Steegstra, O. Nur, Q-H. Hu, M. M. Rahman, M.Willander and R. Turan, Proceedings: "ENS 2007, Paris: France (2007)".

[10] ZHAO Bo, LI-Q-S, Qi H-X and ZHANG N, Chin Phys.Lett., 23 (2006) 1299.

[11] R. G. Singh, Fouran Singh, V. Aggarwal and R. M. Mehra, J.Phys. D: Appl. Phys., 40 (2007) 3090.

[12] Yu-Yun Peng, Tsung-Eong Hseih and Chia-Hung Hsu, Nanotechnology, 17 (2006) 174.

[13] U. Pal, J. Garcia Serrano, N. Koshizaki and T. Sasaki, Mater. Sci. Eng. B, 113 (2004) 24.

[14] Yu-Yun Peng, Tsung-Eong Hseih and Chia-Hung Hsu, App.Phys.Lett., 89 (2006) 211909.

[15] M.Bouguerra, M.Samah, M.A.Belkhir, A.Chergui, L.Gerbous, G.Nouet, D.Chateigner and R.Madelon, Chem. Phys. Lett., 425 (2006) 77.

[16] S.Chakrabarti, D.Ganguly and S Chaudhuri, J.Phys. D:Appl. Phys.,36 (2003) 146.

[17] Mikrajuddin, F.Iskandar, K.Okuyama and F.G.Shi, J.Appl.Phys., Vol. 89, No. 11 (2001) 6431.

[18] S.Chakrabarti, D.Das, D.Ganguly and S.Chaudhuri, Thin Solid Films,441 (2003) 228.

[19] Z.Fu, B.Yang, Lin Li, W.Dong, C.Jia and W.Wu, J.Phys.:Condens. Mater, 15 (2003) 2867.

[20] J.G.Ma, Y.C.Liu, C.S.Xu, Y.X.Liu, C.L.Shao, H.Y.Xu, J.Y.Zhang, Y.M.Lu,D.Z.Shen and X.W.Fan, J. Appl. Phys., 97 (2005) 103509.

[21] Z.D.Sha, Y.Yan, W.X.Qin, X.M.Wu and L.J.Zhuge, J.Phys. D:Appl. Phys.,39 (2006) 3240.

[22] U.Pal, N.Koshizaki, Shin-ya Terauchi and T.Sasaki, Microsc. Microanal. Microstruct, 8 (1997) 403.

[23] G.Mayer, M.Fonin, U.Rudiger, R.Schneider, D.Gerthsen, N.Janben and R. Bratschitsch, Nanotechnology, 20 (2009) 075601.

[24] Y.Zhu, H.Wang and P.P.Ong, J.Phys.:Condens. Mater, 13 (2001) 787.

[25] B.Yao, H.Shi, H.Bi and L.Zhang, J.Phys.:Condens. Matter, 12 (2000) 6265. 
[26] Y.L.Liu, Y.C.Liu, H.Yang, W.B.Wang, J.G.Ma, J.Y.Zhang, Y.M.Lu, D.Z.Shen and X.W.Fan, J.Phys.D:Appl.Phys., 36 (2003) 2705.

[27] J.M.Yuk, J.Y.Lee, J.H.Jung, D.U.Lee, T.W.Kim, D.I.Son and W.K.Choi, J.App. Phys., 103 (2008) 083520 .

[28] L.Fernandez, N.Garro, J.Haskouri, M.Perez-Cabero, J.Alvarez-Rodriguez, J.Latorre, C.Guillem, A.Beltrain, D.Beltran and P.Amoros, Nanotechnology, 19 (2008) 225603.

[29] C.Bouvy, W.Marine and B.Su, Chem. Phys. Lett., (2005), doi:10.1016/j.cplett.2007.02.061.

[30] Shabnam Siddiqui, Chhaya Ravi Kant, P. Arun and N.C. Mehra,Phys. Lett. A, 372 (2008) 7068.

[31] Shabnam, Chhaya Ravi Kant and P. Arun, Mater. Res. Bull. (2010), doi:10.1016/j.materresbull.2010.06.049.

[32] LI Hong-Dong, LU Hang, SANG Dn-Dan, LI Dong-Mei, LI Bo, LU Xian-Yi and ZOU Guang-Tian, Chin. Phys. Lett., Vol.25, No. 10 (2008) 3794.

[33] B.D.Yao, Y.F.Chan and N.Wang, App. Phys. Lett., Vol.81, No.4 (2002) 757.

[34] K.Kongjai, D.Srisongrach, N.Mangkorntong, P.Mangkorntong and S.Choopun, CMU.J.Nat.Sci., Vol.7, No. 1 (2008) 37.

[35] S.A.stepanov, E.A. Kondrashkina, R.Kohler, D.V.Novikov, G.Materlik and S.M.Durbin, Phys.Rev.B, Vol.57, No.8 (1998) 4829.

[36] B.K.Tanner, T.P.A.Hase, T.A.Lafford and M.S.Goorsky, JCPDS-international Centre for Diffraction Data 2004, Advances in X-Ray Analysis, Vol.47 (2004) 309.

[37] Pulak Dutta, Current Science, Vol.78 No.12 (2000) 1478.

[38] D.W.Breiby, O.Bunk, J.W.Andreasen, H.T.Lemke and M.M.Nielsen, J.Appl.Cryst., Vol.41 (2008) 262.

[39] Gwo-Mei Wu and Chen-Yen Wu, Cryst. Res. Technol., 42 (2007) 1271.

[40] C. D Wagner, W. M Riggs, L. E Davis, J. F Moulder and G. E Muilenberg, "Handbook of X-ray photoelectron spectroscopy", Perkin-Elmer Corporation, Physical Electronics Division (1978).

[41] D. Briggs, "Handbook of X-ray and Ultra-violet Photon-electron Spectroscopy", Heyden (London, 1977).

[42] J.Liqiang, Y.Fulong, H.Haige, X.Baifu, C.Weimin and F. Honggang, Sci in China Ser.B Chem. Vol.48 No.1 (2005) 25.

[43] F.Ren, C.Z.Jiang and X.H.Xiao, Nanotechnology, 18 (2007) 285609.

[44] X.H.Wang, S.Liu, P.Chang and Y.Tang, Chin.J.Chem.Phys., Vol.20 No.6, (2007) 632.

[45] “Elements of X-Ray Diffraction” B.D.Cullity (London,1959).

[46] FU Zhu-xi, Guo chang-xin, LIN Bi-xia and LIAO Gui-hong, Chin.Phys.Lett., Vol.15 No.6, (1998) 457.

[47] C.X.Xu, X.W.Sun, B.J.Chen, P.Shum, S.Li and X.Hu, J.Appl.Phys.,Vol.95, No.2 (2004) 661.

[48] Ramon Cusco, Esther Alarcon-Llado, Jordi Ibanez, Luis Artus, Juan Jimenez, Buguo Wang, and Michael J. Callahan, Phys. Rev. B 75 2007) 165202. 
[49] K.A.Alim, V.A.Fonoberov and A.A.Balandin, J.Appl.Phys., 97 (2005) 124313.

[50] T. C. Damen, S. P. S. Porto and B. Tell, Phys. Rev. 142(1966) 570.

[51] M. M. Khayyat, Godsway K. Banini, D. G. Hasko and M. M.Chaudhri, J. Phys. D: Appl Phys., 36 (2003) 1300.

[52] Y. He, L. Bi, J. Y. Feng and Q. L. Wu, J. Phy D: Appl.Phys., 39 (2006) 449.

[53] Chandan Biswas, S. N. Ghosh and C. S. Solanki, Proceedings: "Advances in Energy Research (2006)".

[54] F.H.Leiter, H.R.Alves, A.Hofstaetter, D.M.Hofmann and B.K.Meyer, Phys.Stat.Sol., 226, No.1 (2005) R4-R5.

[55] S.A.Studenikin, Nickolay Golego and Michael Cocivera, J.Appl.Phys., 84, No.4 (1998) 2287.

[56] R. B. M Cross, M. M. De Souza and E. M. Sankara Narayanan, Nanotechnology 16 (2005) 2188. 

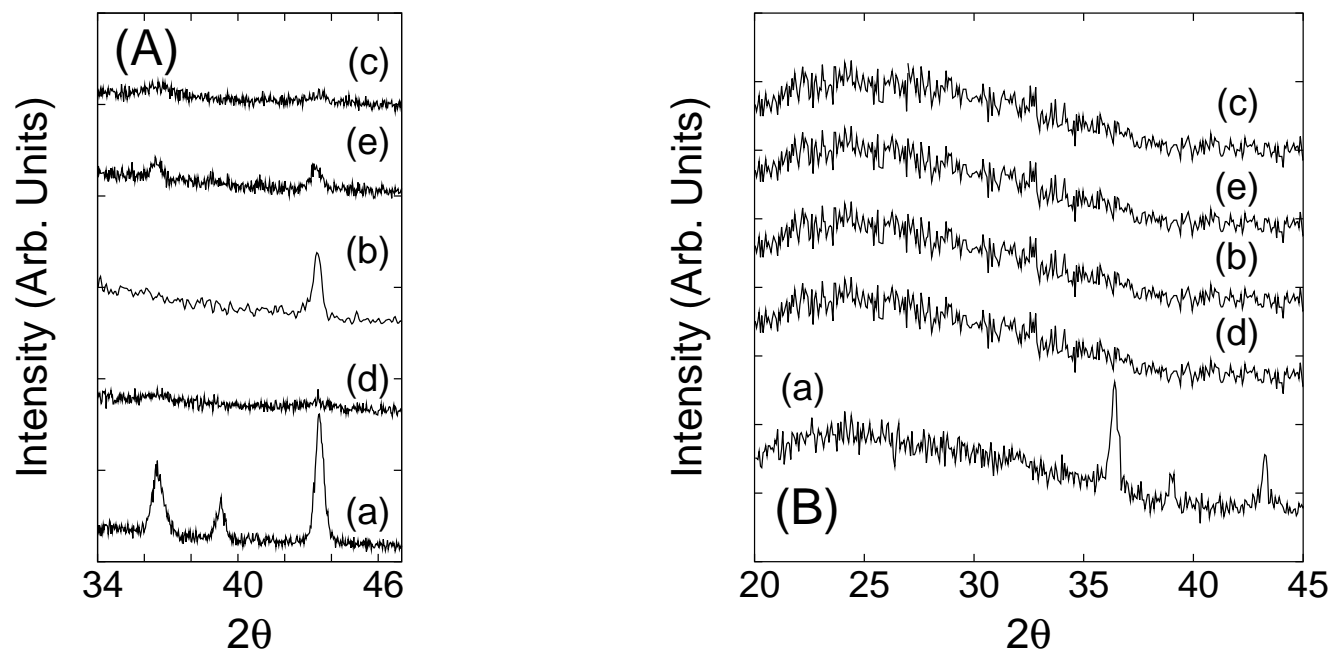

Figure 1: X-Ray Diffractograms of sample (a),(b),(c),(d) and (e) obtained by (A) GIXD and (B) $\theta$-2 $\theta$ technique. 

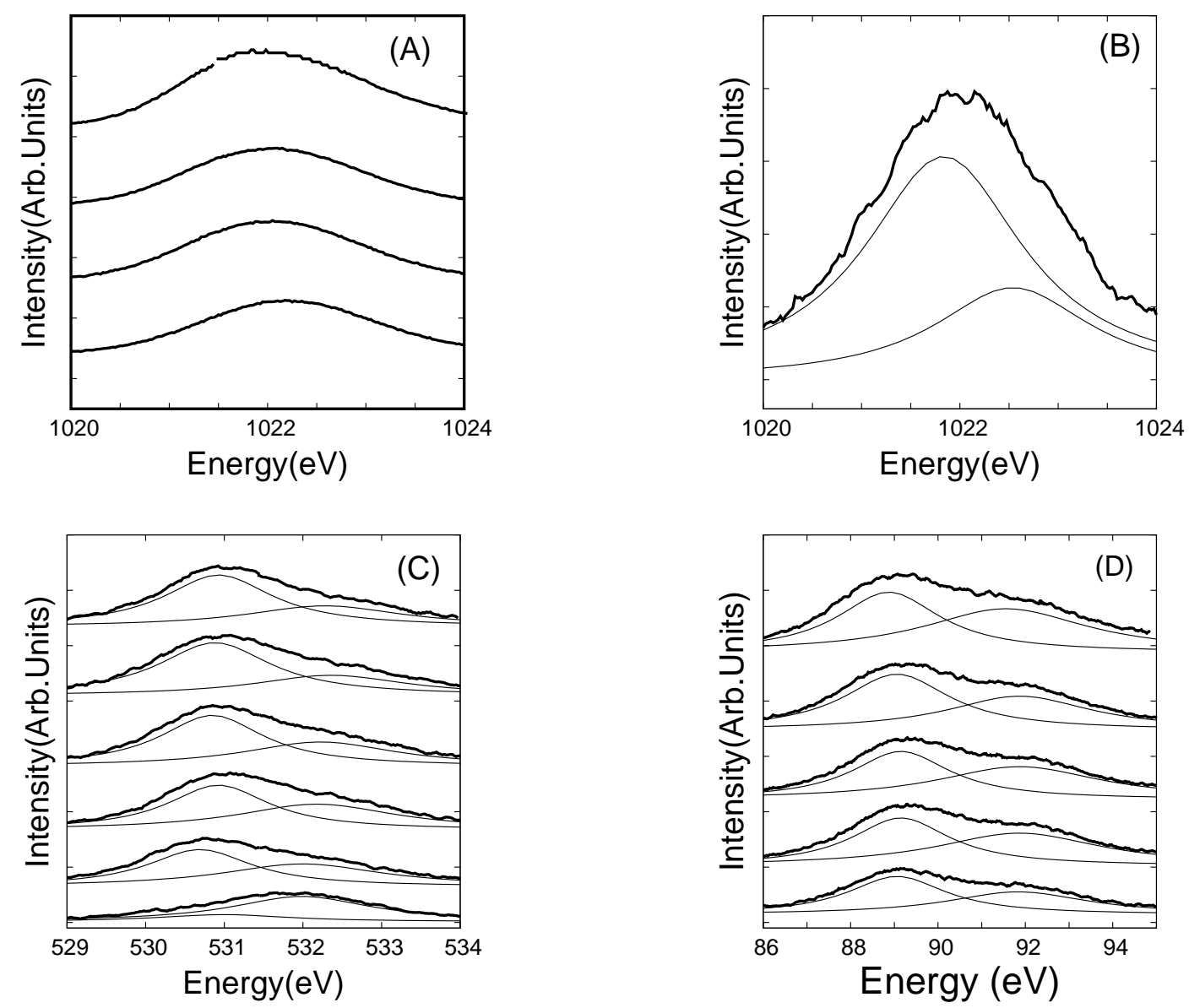

Figure 2: (A) XPS 2 $\mathrm{p}_{3 / 2}$ peaks of Zinc taken at various depth of same sample showing two species, (B) Surface scan, (C) Depth profile scans for Oxygen and (D) Silicon. 

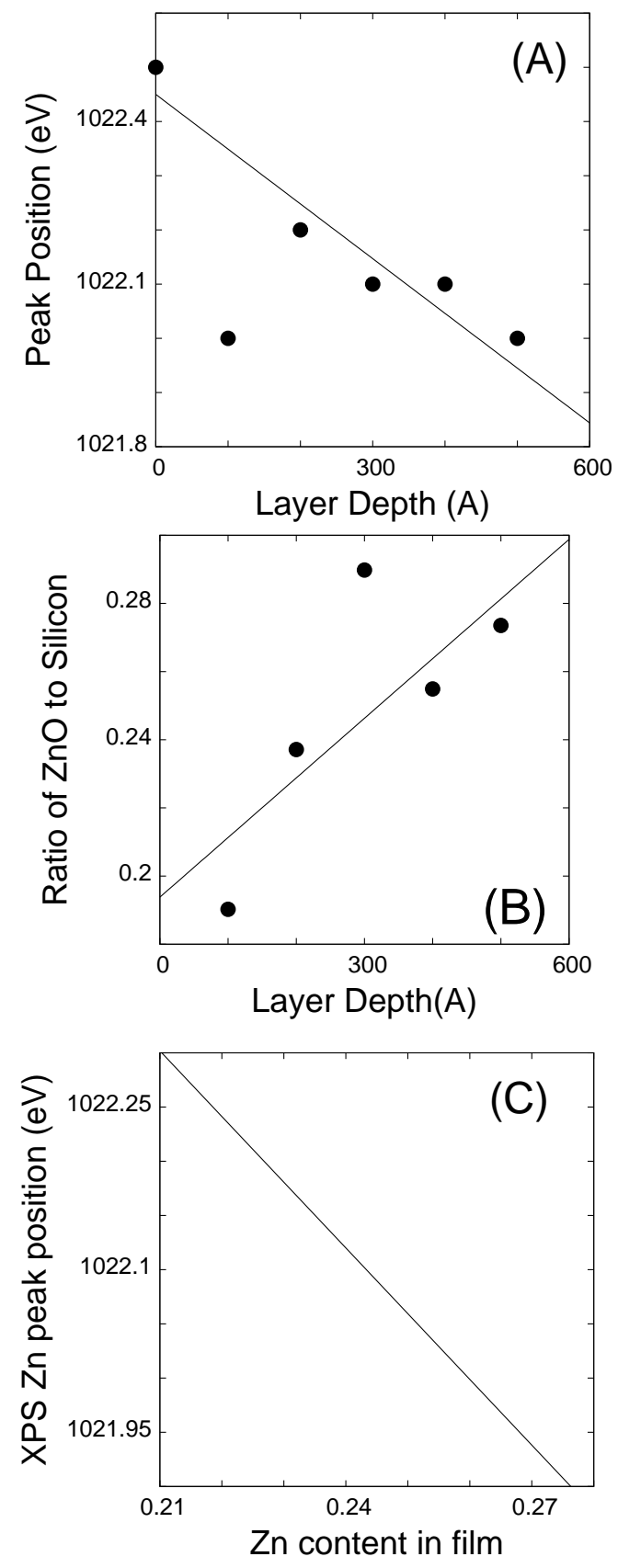

Figure 3: Variation of (A) Peak position of Zn with depth (B) Fraction of Zinc in bonding with Oxygen to amount of Silicon present along the thickness and $(C)$ Peak position of Zinc in bonding with Oxygen to its fraction of presence. 


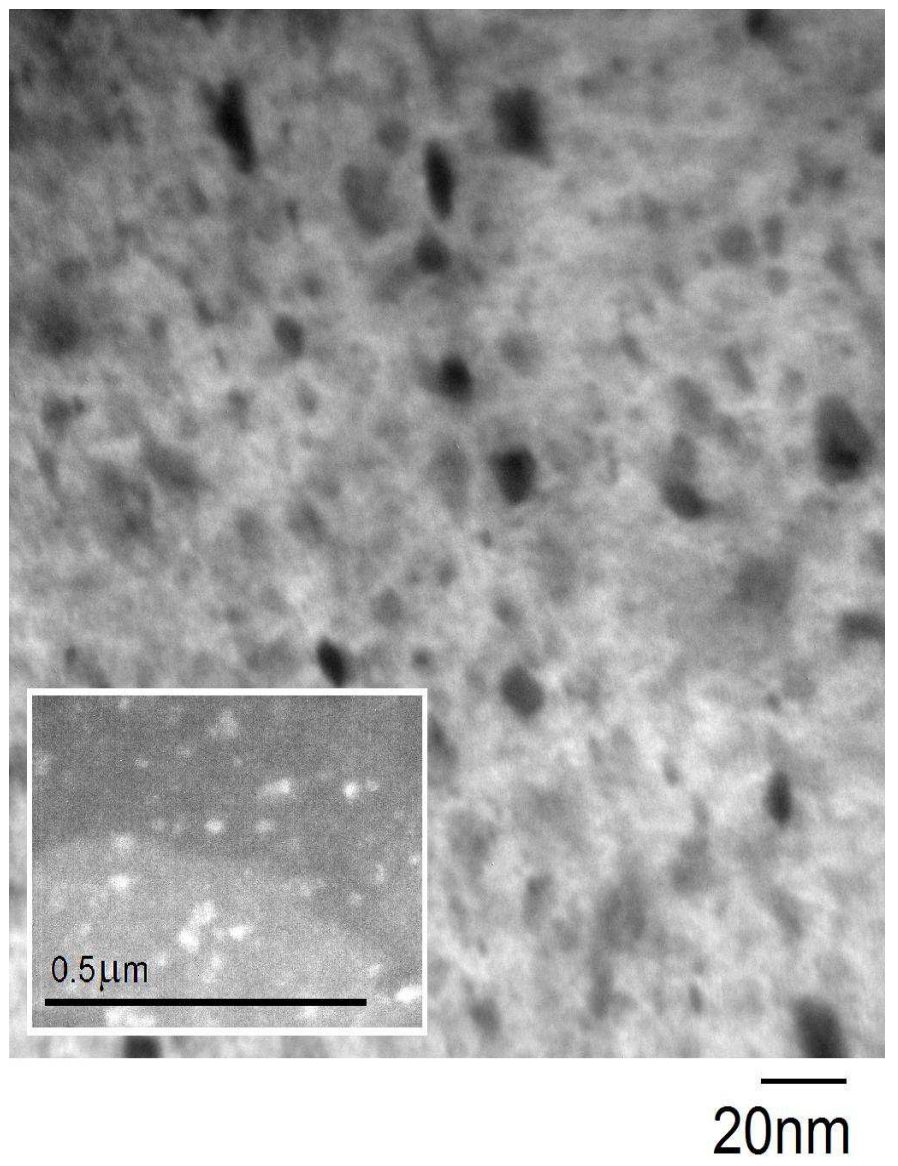

Figure 4: TEM of sample (c). Inset shows dark field image of same sample taken at low magnification which clearly shows formation of two phase with $\mathrm{ZnO}$ clusters embedded in amorphous silicon. 

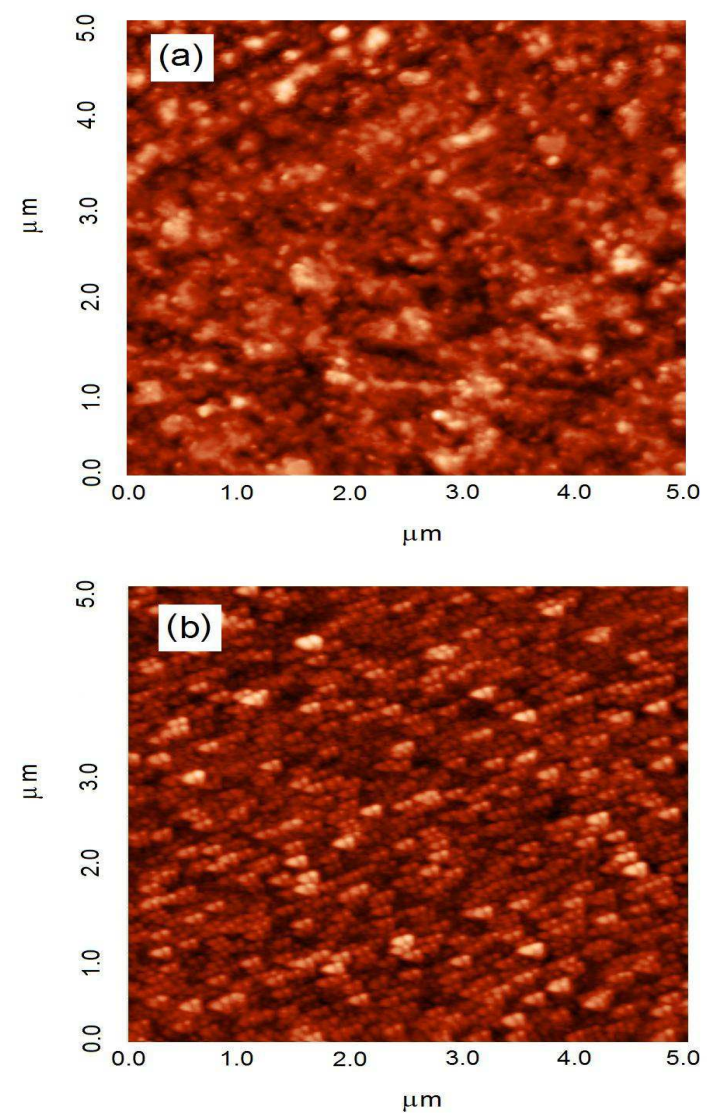

Figure 5: AFM images of sample (a) and sample (b). 

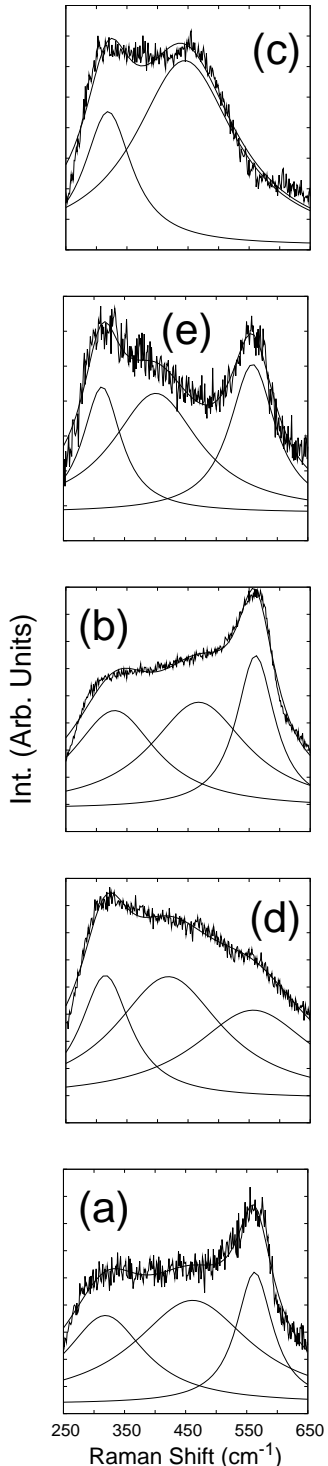

Figure 6: Raman spectra of sample (a), (d), (b), (e) and (c). Also seen are deconvoluted peaks assigned to amorphous silicon, wurtzite structure $\mathrm{ZnO}$ and with oxygen vacancies defects. 


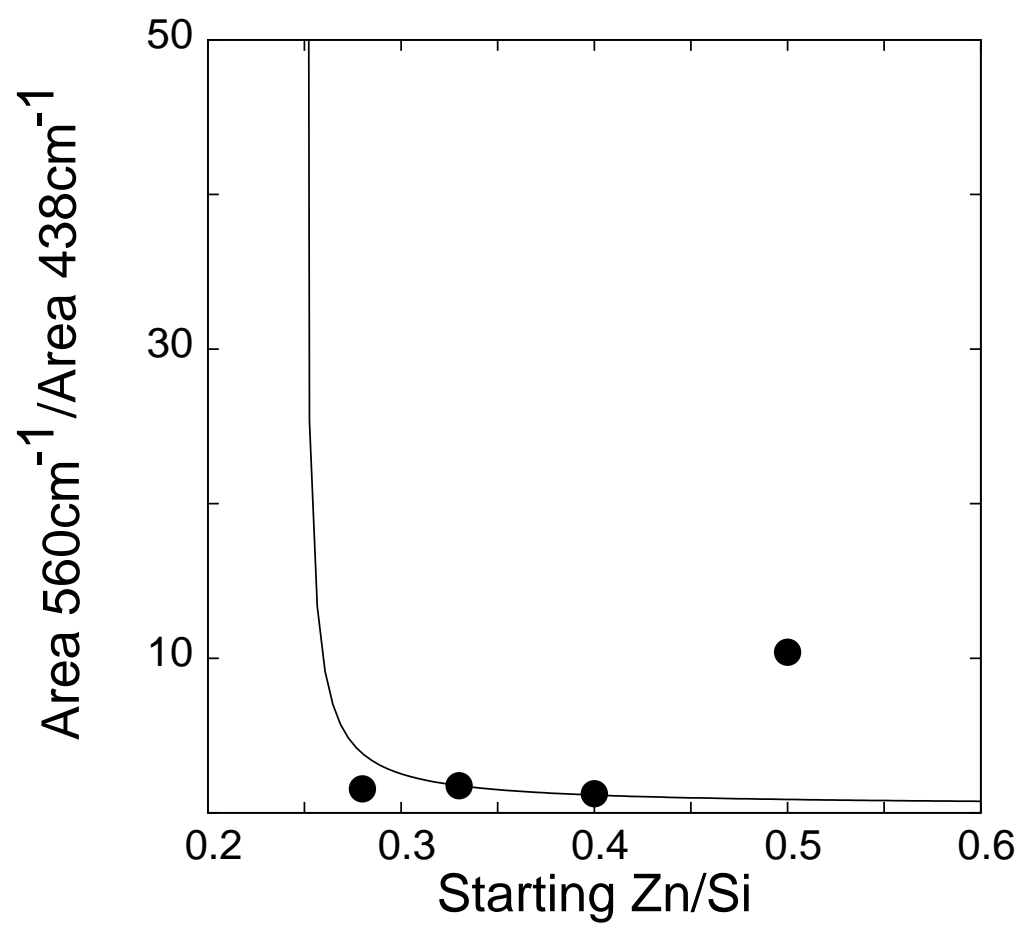

Figure 7: Relative presence of $\mathrm{ZnO}$ with oxygen vacancies to wurtzite structure $\mathrm{ZnO}$ (Area $560 \mathrm{~cm}^{-1} /$ Area $438 \mathrm{~cm}^{-1}$ from Raman spectra) for varying $\mathrm{ZnO}$ content in film. 

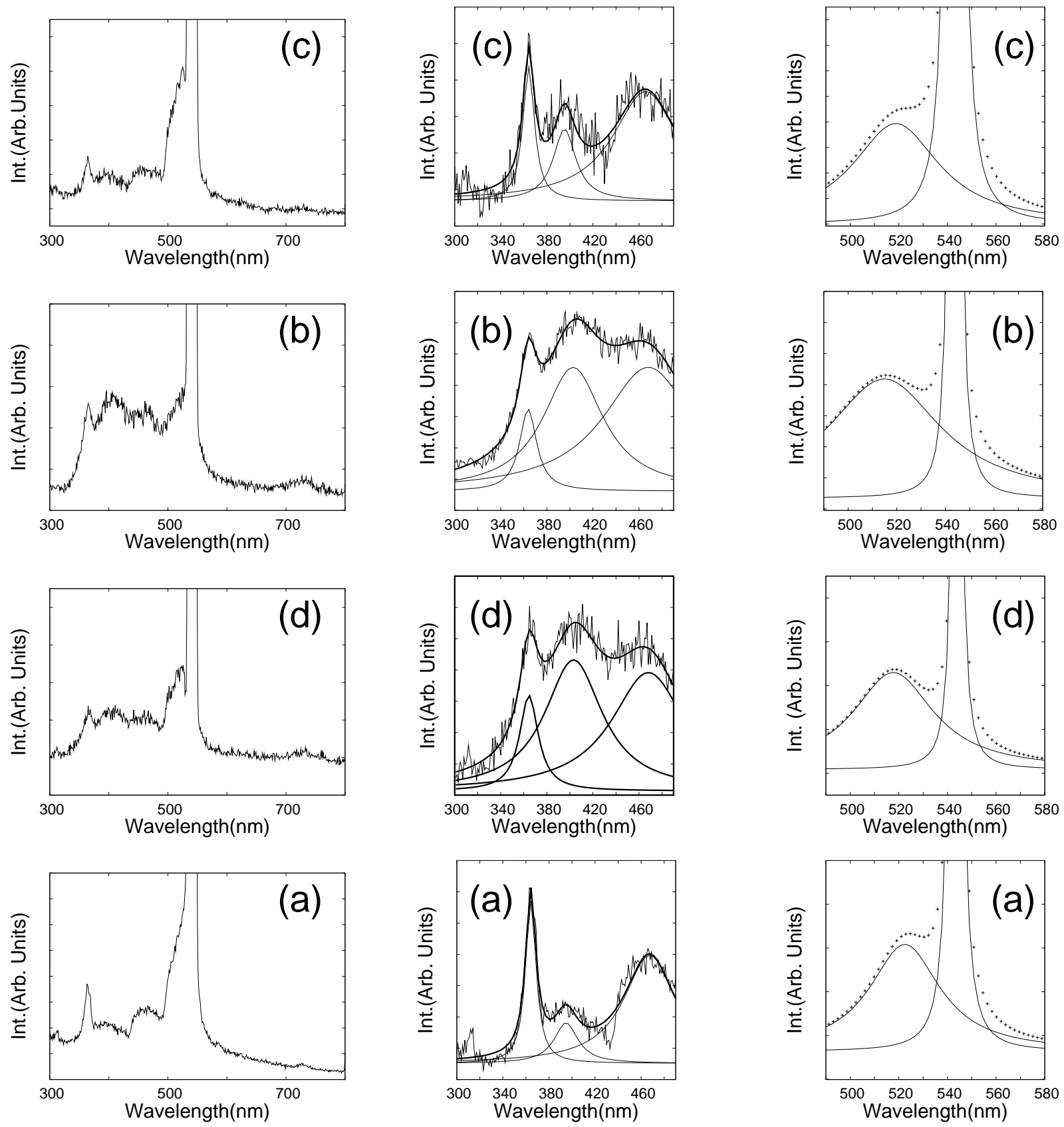

Figure 8: PL of sample (a), (d), (b), and (c). Alongside the raw spectra are shown, deconvolution give 365, 400 and $465 \mathrm{~nm}$ between 300 and 480nm. Also green emission due to defects have been separated from $2^{\text {nd }}$ harmonic to show relative contributions. 

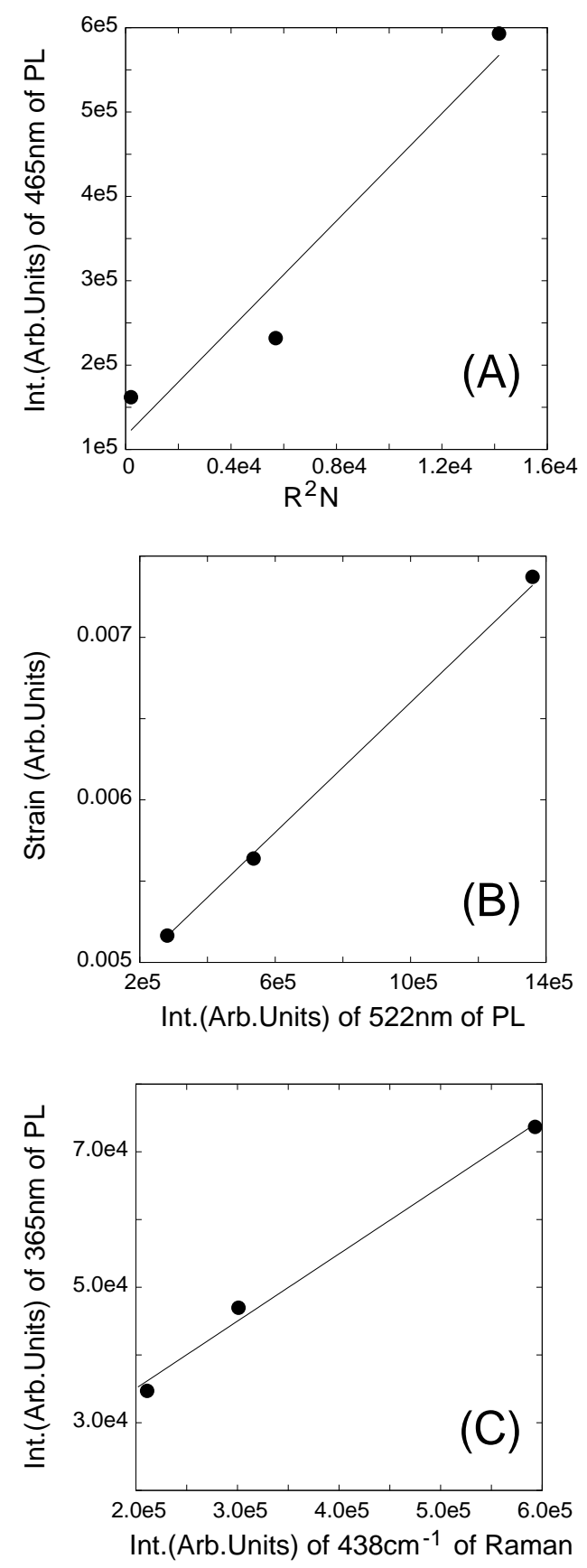

Figure 9: The (A) increase in contribution of $465 \mathrm{~nm} P L$ peak with increasing $\mathrm{R}^{2} \mathrm{~N},(B)$ linear relation in green $(522 \mathrm{~nm})$ emission with strain in film and inturn oxygen vacancies and $(C)$ co-relation in existence of wurtzite peak $\left(438 \mathrm{~cm}^{-1}\right.$ in Raman spectra) and blue emision (365nm peak of $\left.P L\right)$ in samples. 


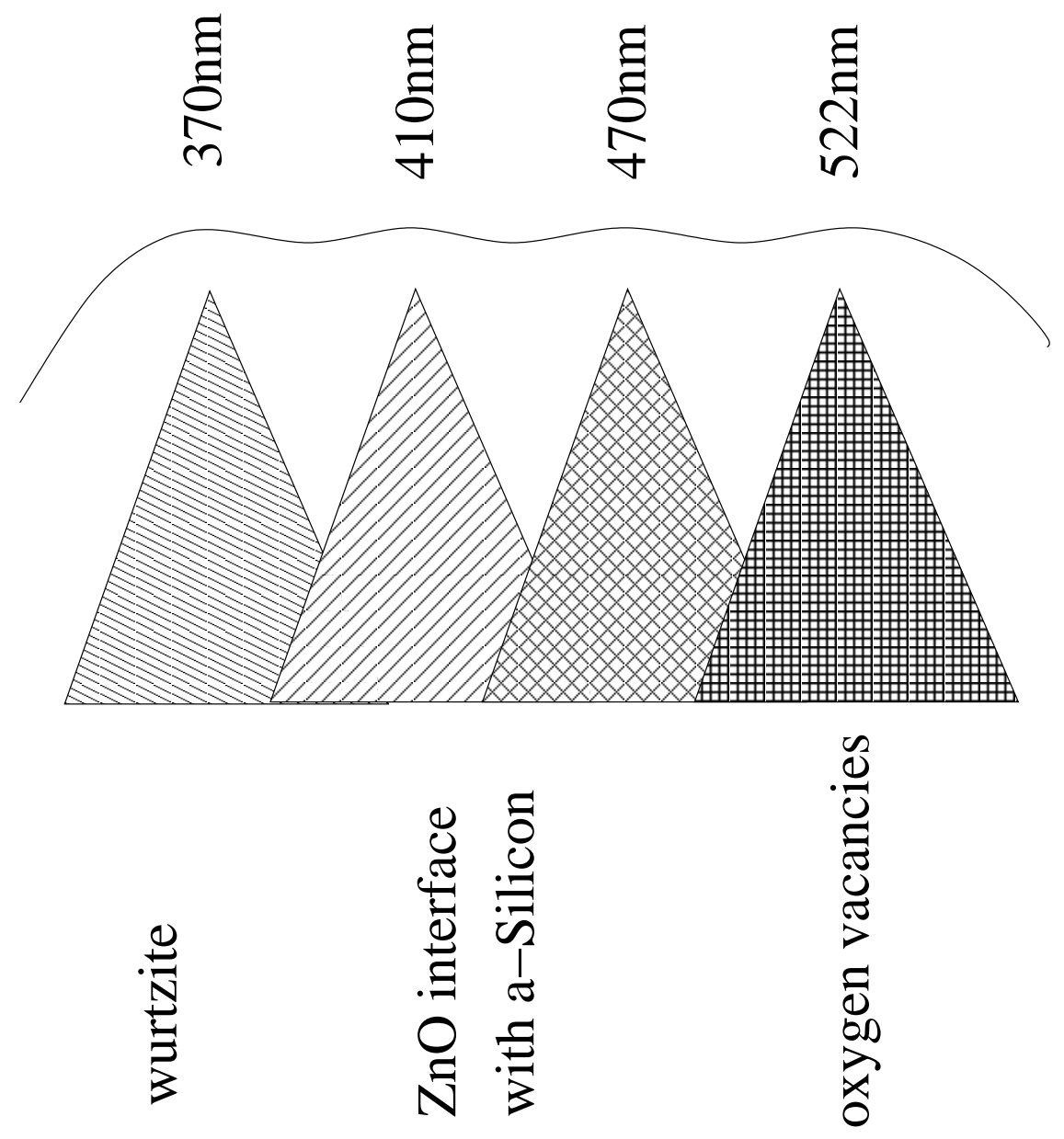

Figure 10: Schematic explaining broadening of PL in $\mathrm{ZnO}$ :Si nanocomposites and their individual contributions.. 